\title{
ON AN INEQUALITY OF FRIEDRICH'S TYPE
}

\author{
MILUTIN R. DOSTANIĆ
}

(Communicated by Palle E. T. Jorgensen)

\begin{abstract}
In this paper the dependence of the constant $C$ in the inequality $\int_{G}|u|^{2} d x \leq C \int_{D}|\nabla u|^{2} d x,\left.u\right|_{\partial D}=0$ on simply connected bounded domains $G \subset D \subset R^{2}$ is found.
\end{abstract}

\section{INTRODUCTION}

It is well known that the inequality

$$
\int_{D}|u|^{2} d x \leq C \int_{D}|\nabla u|^{2} d x \text { (Friedrich's inequality) }
$$

holds, where the function $u$ satisfies the following conditions:

$$
\begin{aligned}
& u \in C^{1}(\bar{D}), \\
& \left.u\right|_{\partial D}=0,
\end{aligned}
$$

and $D$ is a domain in $R^{n}$. The constant $C$ depends only on a domain $D$. Sometimes inequalities of this type are called Poincaré inequalities and sometimes the name of Nirenberg is included.

Inequalities of the form (1) have received considerable attention in the literature because of their fundamental role in the theory of partial differential equations and various applications. For details, we refer to the books by Courant and Hilbert [2], Friedman [4], Ladyzhenskaya and Ural'tseva [5], S.G. Mihlin [6]. In this paper we consider the case $n=2$ i.e. $D \subset R^{2}$. In that case, the best possible constant $C$ in (1) is $\frac{1}{\lambda_{1}(D)}$ where $\lambda_{1}(D)$ is the smallest eigenvalue of the boundary value problem

$$
\begin{aligned}
& -\Delta u=\lambda u, \\
& \left.u\right|_{\partial D}=0 .
\end{aligned}
$$

In some situations we need to estimate the integral $\int_{G}|u|^{2} d x$ in terms of $\int_{D}|\nabla u|^{2} d x$, where $G \subset D \subset R^{2}$ is a simply connected domain. The questions arises in which way the constant $C_{1}$ in

$$
\left\{\begin{array}{l}
\int_{G}|u|^{2} d x \leq C_{1} \int_{D}|\nabla u|^{2} d x \\
u \in C^{1}(\bar{D}),\left.\quad u\right|_{\partial D}=0
\end{array}\right.
$$

depends on the size of $G$.

Received by the editors November 29, 1995 and, in revised form, February 7, 1996.

1991 Mathematics Subject Classification. Primary 47A30.

Key words and phrases. Boundary value problems, Cauchy operator.

(C)1997 American Mathematical Society 


\section{Results}

Let $G$ and $D$ be bounded simply connected domains in $R^{2}$ with piecewise smooth boundaries and $G \subset D$.

Theorem. If $u \in C^{1}(\bar{D})$ and $\left.u\right|_{\partial D}=0$ then the inequality

$$
\int_{G}|u|^{2} d x \leq\left(\lambda_{1}(D) \lambda_{1}(G)\right)^{-\frac{1}{2}} \int_{D}|\nabla u|^{2} d x
$$

holds $\left(\lambda_{1}(D)\right.$ and $\lambda_{1}(G)$ are the smallest eigenvalues of the boundary value problems

$$
\begin{aligned}
-\Delta u & =\lambda u, \quad \text { and }\left.\quad v\right|_{\partial G}=0, \\
\left.u\right|_{\partial D} & =0,
\end{aligned}
$$

respectively) .

Proof. To prove this theorem we need the following lemma from [3]

Lemma. Let $f \in L^{2}(D)\left(D \subset R^{2}\right.$ is a bounded simply connected domain $)$ and

$$
\widehat{f}(x)=\frac{1}{2 \pi} \int_{D} e^{-i u x_{1}-i v x_{2}} f(u, v) d u d v, \quad x=\left(x_{1}, x_{2}\right),
$$

then for $0<\alpha \leq 1 / 2$

$$
\int_{R^{2}} \frac{|\widehat{f}(x)|^{2}}{|x|^{2 \alpha}} d x \leq \lambda_{1}^{-\alpha} \int_{D}|f(x)|^{2} d x
$$

where $\lambda_{1}$ is the smallest eigenvalue of the boundary value problem

$$
\begin{aligned}
& -\Delta u=\lambda u, \\
& \left.u\right|_{\partial D}=0 .
\end{aligned}
$$

Consider now the Cauchy integral operator $T: L^{2}(D) \rightarrow L^{2}(G)$ defined by

$$
\begin{array}{r}
T f(z)=-\frac{1}{\pi} \int_{D} \frac{f(\xi)}{\xi-z} d A(\xi), \\
d A(\xi)=d p d q, \xi=p+i q .
\end{array}
$$

If $\varphi \in C_{0}^{\infty}(D), \psi \in C_{0}^{\infty}(G)$, then (because $\widehat{\left(\frac{1}{z}\right)}=\frac{i}{z}$ in the sense of distributions)

$$
(T \varphi, \psi)=\frac{2}{i} \int_{C} \frac{\hat{\varphi}(z) \overline{\hat{\psi}(z)}}{z} d A(z)
$$

Applying the Lemma (case $\alpha=\frac{1}{2}$ ) we obtain

$$
\begin{aligned}
\left|\int_{C} \frac{\hat{\varphi}(z) \overline{\hat{\psi}(z)}}{z} d A(z)\right| & \leq\left(\int_{C} \frac{|\hat{\varphi}|^{2}}{|z|} d A(z)\right)^{\frac{1}{2}}\left(\int_{C} \frac{\hat{\psi}^{2}}{|z|} d A(z)\right)^{\frac{1}{2}} \\
& \leq\left(\lambda_{1}(D) \lambda_{1}(G)\right)^{-\frac{1}{4}}\|\varphi\|_{L^{2}(D)}\|\psi\|_{L^{2}(G)} .
\end{aligned}
$$

From this inequality and (2) we get

So

$$
\left|(T \varphi, \psi)_{L^{2}(G)}\right| \leq\left(\lambda_{1}(D) \lambda_{1}(G)\right)^{-\frac{1}{4}}\|\varphi\|_{L^{2}(D)}\|\psi\|_{L^{2}(G)} .
$$

$$
\|T\| \leq 2\left(\lambda_{1}(D) \lambda_{1}(G)\right)^{-\frac{1}{4}},
$$


i.e.

$$
\int_{G}|T f|^{2} d A \leq 4\left(\lambda_{1}(D) \lambda_{1}(G)\right)^{-\frac{1}{2}} \int_{D}|f|^{2} d A
$$

for every $f \in L^{2}(D)$.

Let $u$ be a real function such that $u \in C^{1}(\bar{D})$ and $\left.u\right|_{\partial D}=0$. By the CauchyGreen formula [7] we have

$$
u(z)=\frac{1}{2 \pi i} \int_{\partial D} \frac{u(\xi)}{\xi-z} d \xi+T\left(\frac{\partial u}{\partial \bar{z}}\right), z \in G,
$$

i.e.

$$
u(z)=T\left(\frac{\partial u}{\partial \bar{z}}\right)
$$

because $\left.u\right|_{\partial D}=0$.

From (3) and (4) we get

$$
\int_{G}\left|T \frac{\partial u}{\partial \bar{z}}\right|^{2} d A(z) \leq 4\left(\lambda_{1}(D) \lambda_{1}(G)\right)^{-\frac{1}{2}} \int_{D}\left|\frac{\partial u}{\partial \bar{z}}\right|^{2} d A(z),
$$

i.e.

$$
\int_{G}|u|^{2} d A \leq 4\left(\lambda_{1}(D) \lambda_{1}(G)\right)^{-\frac{1}{2}} \int_{D}\left|\frac{\partial u}{\partial \bar{z}}\right|^{2} d A
$$

i.e.

$$
\int_{G}|u(z)|^{2} d A(z) \leq\left(\lambda_{1}(D) \lambda_{1}(G)\right)^{-\frac{1}{2}} \int_{D}|\nabla u|^{2} d A(z)
$$

because

$$
\frac{\partial u}{\partial \bar{z}}=\frac{1}{2}\left(\frac{\partial u}{\partial x}+i \frac{\partial u}{\partial y}\right)
$$

Remark 1. The constant $\left(\lambda_{1}(D) \lambda_{1}(G)\right)^{-\frac{1}{2}}$ is the best possible one in the sense that there exists a smooth function $u_{0}$ such that

$$
\sqrt{\lambda_{1}(G)} \int_{G}\left|u_{0}(z)\right|^{2} d A(z) \rightarrow\left(\lambda_{1}(D)\right)^{-\frac{1}{2}} \int_{D}\left|\nabla u_{0}\right|^{2} d A(z)
$$

if the domain $G$ tends to the domain $D$.

Indeed, for $G_{r}\left(G_{r}=\varphi\left(u_{r}\right), u_{r}=\{z:|z|<r<1\}, \varphi\right.$ is a conformal mapping of the unit disc $\{z:|z|<1\}$ on $D)$ we have $\lambda_{1}\left(G_{r}\right) \rightarrow \lambda_{1}(D)$ when $r \rightarrow 1$. (That follows from min-max principle.)

If $u_{0}$ is the eigenfunction associated with the first eigenvalue of the problem

$$
\begin{aligned}
& -\Delta u=\lambda u, \\
& \left.u\right|_{\partial D}=0,
\end{aligned}
$$

then

$$
\begin{gathered}
\sqrt{\lambda_{1}(G)} \int_{G}\left|u_{0}(z)\right|^{2} d A(z) \longrightarrow \sqrt{\lambda_{1}(D)} \int_{D}\left|u_{0}\right|^{2} d A(z) \\
=\left(\lambda_{1}(D)\right)^{-\frac{1}{2}} \int_{D}\left|\nabla u_{0}\right|^{2} d A(z)
\end{gathered}
$$

when $r \rightarrow 1$ because $-\Delta u_{0}=\lambda_{1}(D) u_{0}$ and $\left.u_{0}\right|_{\partial D}=0$. 
Remark 2. Using Faber-Krahn inequality [1]

$$
\lambda_{1}(G) \geq \frac{\pi j_{0}^{2}}{|G|} ; \quad \lambda_{1}(D) \geq \frac{\pi j_{0}^{2}}{|D|},
$$

$j_{0}$ is smallest positive zero of the Bessel function $J_{0},|G|,|D|$ denote the area of $G$ and $D$ respectively, we get a more rough but more operative inequality

$$
\int_{G}|u|^{2} d A(z) \leq \frac{\sqrt{|D||G|}}{\pi j_{0}^{2}} \int_{D}|\nabla u|^{2} d A(z) .
$$

\section{ACKNOWLEDGMENT}

The author is grateful to the referee for useful remarks.

\section{REFERENCES}

1. C. Bandle, Isoperimetric inequalities and applications, Pitman, London, 1980. MR 81e:35095

2. R. Courant and D. Hilbert, Methods of Mathematical Physics Vol. 1, Wiley, New York, 1953. MR 16:426a

3. M. Dostanić, The properties of the Cauchy transform on a bounded domain, Journal of Operator Theory (to appear).

4. A. Friedman, Partial differential equations of parabolic type, Prentice Hall, Englewood Cliffs N. Y., 1964. MR 31:6062

5. O. A. Ladyzhenskaya and N. N. Uralt'seva, Linear and Quasilinear elliptic equations, Academic Press, New York, 1969.

6. S. G. Mihlin, Lectures on Mathematical Physics, Moscow, 1968.

7. I.N. Vekua, Generalized analytic functions, Moscow, 1988. MR 90a:30135

Matematicki Fakultet, Studentski trg 16, Beograd, Serbia 